\title{
Correction: "Bo Zhou, Erlin Meng, Zhenqian Chen, Synthesis of Porous Carbon Sphere Based on Starch and Its Application of Electric Double Layer Capacitor" [Int. J. Electrochem. Sci., 13 (2018) 2441 -2447]
}

\author{
Bo Zhou ${ }^{1,2}$, Erlin Meng ${ }^{1}$, Zhenqian Chen ${ }^{2, *}$ \\ ${ }^{1}$ SUST, School of Environment Science and Engineering, Suzhou University of Science and \\ Technology, Suzhou, 215009, P. R. China \\ ${ }^{2}$ IIUSE, Key Laboratory of Energy Thermal Conversion and Control of Ministry of Education, School \\ of Energy and Environment, Southeast University, Nanjing, 210096, P. R. China
}

5 November 2018

The paper has been corrected at the author's request. Paper entitled" Synthesis of Porous Carbon Sphere Based on Starch and Its Application of Electric Double Layer Capacitor". This paper has been officially published(Int. J. Electrochem. Sci., 13 (2018) 2441 - 2447, doi: 10.20964/2018.03.32). Due to the author named Zhenqian Chen didn't do any work with this paper, Other authors of this paper asked to remove the author's name of Zhenqian Chen in the paper. The corrected name should be "Bo Zhou, Erlin Meng, Synthesis of Porous Carbon Sphere Based on Starch and Its Application of Electric Double Layer Capacitor" (Int. J. Electrochem. Sci., 13 (2018) 2441 -2447 )". All authors of the paper bear all responsibilities and have nothing to do with the International Journal of Electrochemical Science.

(C) 2018 Published by ESG (www.electrochemsci.org). 